\title{
Migration Effects on BBO Evolution in Optimizing Fifteen Element Yagi-Uda Antenna Design
}

\author{
Gagan Sachdeva \\ Rayat Bahra Group of Institutes \\ Mohali Campus, Punjab [INDIA]
}

\author{
Dilpal Singh \\ UIET, Panjab University \\ Chandigarh [INDIA]
}

\begin{abstract}
Biogeography Based Optimization (BBO) is a recently introduced optimization technique based on science of biogeography, i.e., study of distribution of biological species over space and time. In $\mathrm{BBO}$, potential solutions of a problem are grouped in integer vectors known as habitats. Feature, i.e., Suitability Index Variable (SIV), sharing among various habitats is made to occur with migration operator where as exploration of new SIVs is done with mutation operator. Different migration variants are proposed to increase the diversity in the population, with objective of improved performance of BBO algorithm. Yagi-Uda antenna is a widely used antenna design due to various useful properties of high gain, low cost and ease of construction. Designing a Yagi-Uda antenna involves determination of element lengths and spacings between them to get desired radiation characteristics. In this paper, various migration variants of $\mathrm{BBO}$ algorithm, reported till date, are investigated to optimize the lengths and spacings for Yagi-Uda antenna elements for maximum gain. The results obtained with these migration variants are compared and the best results are presented in the ending sections of the paper.
\end{abstract}

\section{Keywords:}

Yagi-Uda Antenna, Biogeography Based Optimization, Antenna Gain, Genetic Algorithm

\section{INTRODUCTION}

Antenna is an electrical device which converts electric signal into free space radiations and vice-versa. The various radiation characteristics that affect the design of an antenna are gain, impedance, bandwidth, frequency of operation, Side Lobe Level (SLL) etc. Yagi-Uda antenna is a widely used directional antenna design due to various desirable features, i.e., high forward gain, low cost and ease of construction. It is basically a parasitic linear array of parallel dipoles, one of which is energized directly by transmission line while the others act as parasitic radiators whose currents are induced by mutual coupling.

Yagi-Uda antenna was invented in 1926 by H. Yagi and S. Uda at Tohoku University [31] in Japan, however, published in English in 1928 [35]. The main objective, in design of Yagi-Uda antenna, is to find an optimum structure that meet certain radiation criteria like gain, impedance, SLL and beamwidth. However, due to its parasitic elements, it is extremely difficult to obtain an optimum design of Yagi-Uda antenna. Since its inception, Yagi- Uda antenna has been optimized several times for gain, impedance, SLL and bandwidth using different optimization techniques based on traditional mathematical approaches $[26,5,9,27,8,7,10]$ and Artificial Intelligence (AI) techniques [16, 34, 33, 4, 18, 30, 29]. In 1949, Fishenden and Wiblin [15] proposed an approximate design of Yagi aerials for maximum gain, however, the approach was based on approximations. In 1959, Ehrenspeck and Poehler proposed a manual approach to maximize the gain of the antenna by varying various lengths and spacings of its elements [14].

Later on, with the availability of high performance computing, it became possible to optimize antennas numerically. Bojsen et al. in [5] proposed an optimization technique to find the maximum gain of Yagi-Uda antenna arrays with equal and unequal spacings between adjacent elements. Cheng et al. in [8] and [7] have used optimum spacings and lengths to optimize the gain of a Yagi-Uda antenna. In [10], Cheng has proposed optimum design of YagiUda antenna where antenna gain function is highly non-linear. The performance of these gradient based techniques depends on choice of initial solution.

In 1975, John Holland introduced Genetic Algorithms (GAs) as a stochastic, swarm based AI technique, inspired from natural evolution of species, to optimize arbitrary systems for certain cost function. Since then many researchers have used GAs to optimize Yagi-Uda antenna designs for gain, impedance and bandwidth separately [1, 16, 11] and collectively [34, 32, 17]. Jones et al. in [16] have used GA to optimize Yagi-Uda antenna for various radiation characteristics and compared the result with steepest gradient method. Baskar et al. in [4], have used Comprehensive Learning Particle Swarm Optimization (CLPSO) to optimize Yagi-Uda antenna and obtained better results than other optimization techniques. In [18], Li has optimized Yagi-Uda antenna using Differential Evolution (DE) and illustrated the capabilities of the proposed method with several Yagi-Uda antenna designs. In [30], Singh et al. have analyzed another useful, stochastic global search and optimization technique known as Simulated Annealing (SA) for the optimization of Yagi-Uda antenna. In 2008, Dan Simon introduced a new optimization technique based on science of biogeography, in which information sharing among various habitats, i.e., potential solutions, is obtained via migration operator and exploration of new features is done with mutation operator [28]. Singh et al. have presented $\mathrm{BBO}$ as a better optimization technique for Yagi-Uda antenna designs, as compared to other optimization techniques in [29]. In [19], Li has proposed the Bi-Swarm optimization technique to optimize the Yagi-Uda antenna and produced better result than GA, Particle Swarm Optimization (PSO) and Computer Intelligence (CI) techniques. In 2011, Amaral et al. has applied Ellipsoid algorithm to optimization of Yagi-Uda antenna for gain maximization [2]. Li et al. in [20] have used Invasive Weed Optimization (IWO) technique to optimize a six element Yagi-Uda antenna for maximum directivity.

In [13], Du et al. have proposed the concept of immigration refusal in BBO aiming at improved performance.In [21], Ma and Simon introduced another migration operator, i.e., Blended mi- 
gration, to solve constrained optimization problems and make BBO convergence faster. In [24], Pattnaik et al. have proposed Enhanced Biogeography Based Optimization (EBBO) in which duplicate habitats, created due to migration of features, are replaced with randomly generated habitats to increase the exploitation ability of $\mathrm{BBO}$ algorithm.

In this paper, various migration variants in $\mathrm{BBO}$ algorithm are experimented to optimize fifteen-element Yagi-Uda antenna to investigate their relative optimization performance. A method of moments based programme, NEC2 (Numerical Electromagnetics Code version 2), is used to evaluate the antenna designs for gain.

After this brief introduction, the paper is structured as follows: In Section 2, Yagi-Uda antenna is briefly discussed. Section 3 is dedicated to biogeography terminology, BBO technique and its migration variants. In Section 4, comparative simulation results obtained with different migration operators, during optimizing antenna designs, are presented. Finally, paper is concluded in Section 5.

\section{ANTENNA DESIGN PARAMETERS}

Yagi-Uda antenna is basically made of three types of elements: (a) Reflector (b) Feeder and (c) Directors. Reflector is longest of all elements and blocks radiations in one direction. Feeder or driven element is fed with the signal to be transmitted, directly from transmission line. Directors are usually more than one in number and are responsible for unidirectional radiations. Normally, there is no limit on number of directors, however, as the number of directors are increased beyond a certain limit there is a reduction in the induced current in the most extreme elements. Figure 1 presents a basic Yagi-Uda antenna design where all elements are placed along $y$-axis and parallel to $x$-axis. Middle segment of the reflector is placed at origin and signal to be transmitted is fed to the middle segment of the feeder element. An incoming field induces resonant currents on all the antenna elements which causes parasitic (reflector and directors) elements to re-radiate signals. These re-radiated fields are then picked up by the feeder element, that makes total current induced in the feeder equivalent to combination of the direct field input and the reradiated contributions from the director and reflector elements.

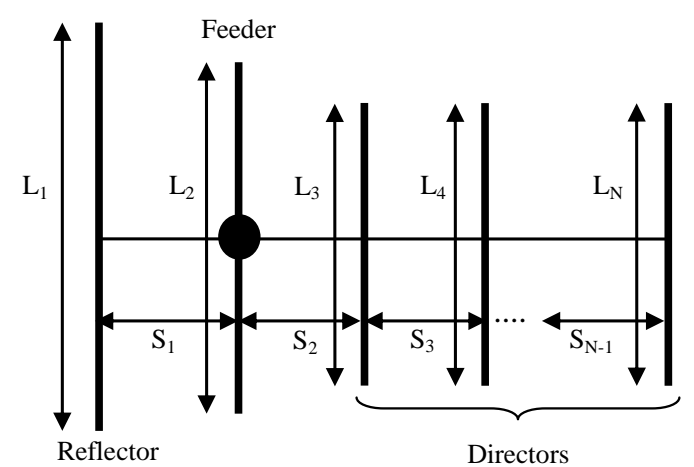

Fig. 1. Basic Yagi-Uda Antenna Design

Element lengths and spacings between them are the variables/parameters which need to be determined for optimum design of Yagi-Uda antenna. An antenna with $N$ elements requires $2 N-1$ parameters, i.e., $N$ wire lengths and $N-1$ spacings, to be determined. These $2 N-1$ parameters, collectively, are represented as an integer vector referred as a habitat in $\mathrm{BBO}$ given

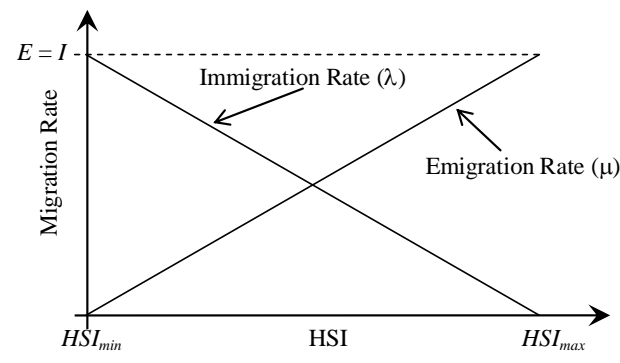

Fig. 2. Migration Curves

as (1).

$$
H=\left[L_{1}, L_{2}, \ldots, L_{N}, S_{1}, S_{2}, \ldots, S_{N-1}\right]
$$

where $L_{N}$ are the lengths and $S_{N-1}$ are the spacings between antenna elements.

\section{BIOGEOGRAPHY BASED OPTIMIZATION}

Biogeography Based Optimization is a population based global optimization technique based on the science of biogeography, i.e., study of the distribution of animals and plants among different habitats over time and space. BBO results presented by researches, to optimize Yagi-Uda antenna, are better than other optimization techniques like PSO, GAs, SA, DE etc. [16, 32] 4, 25].

Initially, biogeography was studied by Alfred Wallace [3] and Charles Darwin [12] mainly as descriptive study. However, in 1967, the work carried out by MacAurthur and Wilson [22] changed this perception by introducing a mathematical model for biogeography which made it possible to predict the number of species in a habitat. Mathematical models of biogeography describe the migration, speciation and extinction of species in various habitats.

A habitat or island is an ecological area inhabited by a particular animal species which is geographically isolated from other habitats. Each habitat is characterized by its Habitat Suitability Index (HSI). Habitats which are well suited as living places for biological species are referred to have high HSI value. HSI is analogues to fitness in other Evolutionary Algorithms whose value is a function of many features of the habitat such as rainfall, diversity of vegetation, diversity of topographic features, land area, and temperature etc. The features/variables that characterize habitability are known as Suitability Index Variables (SIVs). In other words, HSI is dependent variable whereas SIVs are independent variables.

The habitats with high HSI have large probability of emigration (hence high emigration rate, $\mu$ ) simply due to large number of species they host and small probability of immigration (low immigration rate, $\lambda$ ) as they are already saturated with species. Immigration can be defined as the arrival of new species into a habitat, while emigration is the process of leaving one's native habitat. Similarly, habitats with low HSI tend to have low emigration rate, $\mu$, due to sparse population, however, they will have high immigration rate, $\lambda$. Suitability of habitats having low HSI value is likely to increase with more number of species arriving from habitats having high HSI as suitability of a habitat depends upon its biological diversity. For sake of simplicity, it is safe to assume a linear relationship between HSI (or population) and immigration and emigration rates. Also maximum emigration and immigration rates are assumed equal, i.e., $E=I$, as shown graphically in Figure 2. 
For $k$-th habitat, values of emigration rate, $\mu_{k}$, and immigration rate, $\lambda_{k}$, are given by (2) and (3).

$$
\begin{gathered}
\mu_{k}=E \cdot \frac{H S I_{k}}{H S I_{\max }-H S I_{\min }} \\
\lambda_{k}=I \cdot\left(1-\frac{H S I_{k}}{H S I_{\max }-H S I_{\min }}\right)
\end{gathered}
$$

Good solutions (habitats with high HSI) are more resistant to change than poor solutions (habitats with low HSI) whereas poor solutions are more dynamic in nature and accept a lot of new features from good solutions. This addition of new features to low HSI solutions from high HSI solutions may raise the quality of those solutions.

In a global optimization problem with number of possible solutions, each habitat or a solution in a population of size $N P$ is represented by $M$-dimensional integer vector as $H=$ $\left[S I V_{1}, S I V_{2}, \ldots, S I V_{M}\right]$ where $M$ is the number of SIVs (features) to be evolved for optimal HSI. HSI is the fitness criteria that is determined by evaluating the cost/objective function, i.e., $H S I=f(H)$. BBO consists of mainly two mechanisms: (A) Migration and (B) Mutation, these are discussed in the following subsections.

\subsection{Migration}

Migration is a probabilistic operator that improves HSI of poor habitats by sharing information from good habitats. During Migration, immigrating habitat, ImHbt, use its immigration rate, $\lambda$, given by (3), to probabilistically decide whether to immigrate or not. In case immigration is selected, then the emigrating habitat, $E m H b t$, is found probabilistically based on emigration rate, $\mu$, given by (2). The process of migration is completed by copying values of SIVs from EmHbt to ImHbt at random chosen sites. $\mathrm{BBO}$ employing above mentioned migration scheme is termed as the standard BBO. The migration operator may lead to same types of habitats in large number after few iterations. Different migration variants are proposed to increase the diversity in the population, with objective of improved performance of $\mathrm{BBO}$ algorithm, whose pseudo codes are given in Algorithm 1 and explained in following subsections.

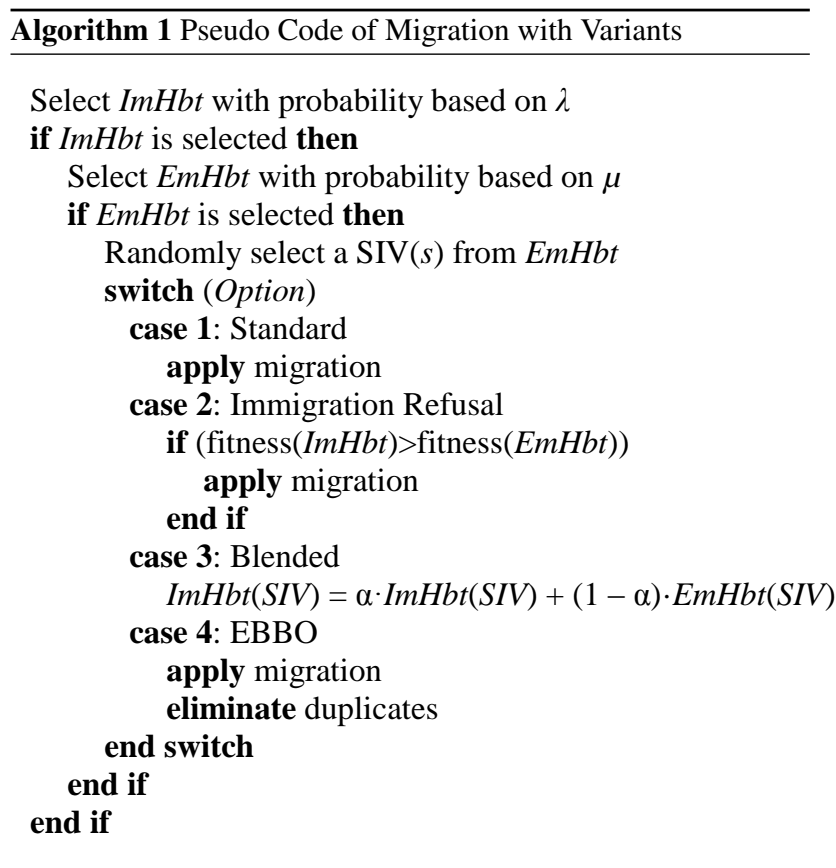

3.1.1 Immigration Refusal BBO (IRBBO). In BBO, a habitat with high emigration rate have high probability of emigration to other habitats and low probability of immigration from other habitats. However, the low immigration probability does not mean that immigration will never happen. Once in a while, a highly fit solution may receive solution features from a low-fit solution that may degrade its fitness. In such cases, immigration is refused to prevent degradation of fitness, i.e., HSI values of highly fit habitats. This $\mathrm{BBO}$ variant with conditional migration is termed as IRBBO and gives good performance with testbed of benchmark functions [13].

3.1.2 Blended Migration. Blended migration operator [21] is a generalization of the standard BBO migration operator which is inspired from blended crossover in GAs [23]. Here, SIVs of immigrating habitat, ImHbt, are not simply replaced by SIVs of emigrating habitat, EmHbt, like standard migration operation. Instead, a new value for $i$-th SIV, $S I V_{\text {new }}$, is comprised of two components given as (4)

$$
S I V_{\text {new }}=\alpha \cdot \operatorname{ImHbt}(S I V)+(1-\alpha) \cdot \operatorname{EmHbt}(S I V)
$$

where $\alpha$ is a random number between 0 and 1 . The pseudo code of blended migration is depicted as case 3 in Algorithm 1.

3.1.3 Enhanced Biogeography Based Optimization. Standard BBO migration operator tends to create duplicate solutions which decreases the diversity in the population. To prevent this diversity decrease, duplicate habitats are replaced with randomly generated habitats, as depicted as case 4 in Algorithm 1. This leads to increase exploration of new SIV values. In EBBO, clear duplicate operator is integrated in basic $\mathrm{BBO}$ to improve its performance [24].

\subsection{Mutation}

Mutation is another probabilistic operator that alters the values of randomly selected SIVs of some habitats that are intended for exploration of search space for better solutions by increasing the biological diversity in the population. Here, higher mutation rates are investigated on habitats those are, probabilistically, participating less in migration process. Elitism approach is generally used along with mutation to preserve features of the best habitat. The mutation rate, mRate, for $k$-th habitat is calculated as (5)

$$
\text { mate }_{k}=C \times \min \left(\mu_{k}, \lambda_{k}\right)
$$

where $\mu_{k}$ and $\lambda_{k}$ are emigration and immigration rates, respectively, given by (2) and (3) corresponding to $H S I_{k}$. Here $C$ is a scaling constant and its value is equal to 1 . The pseudo code of mutation operator is given in Algorithm 2.

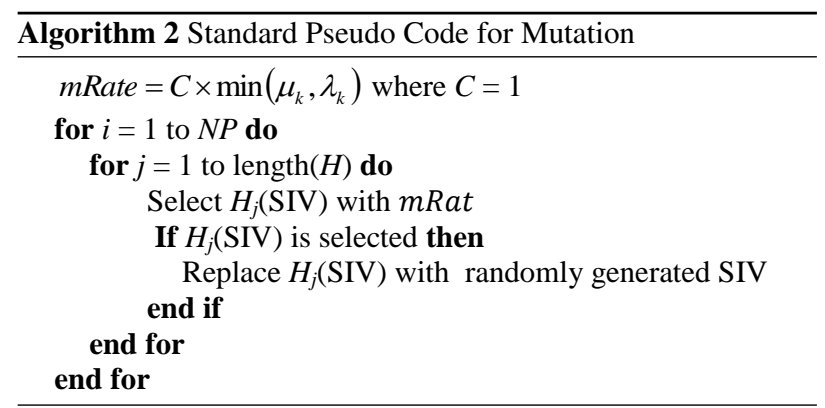

\section{SIMULATION RESULTS AND DISCUSSIONS}

Fifteen-element Yagi-Uda antenna designs are optimized for maximum gain using $\mathrm{BBO}$ with different migration variants, discussed in Section 3.1, and standard mutation operator. Each 
Table 1. Results of Gain Optimized Fifteen-Element Yagi-Uda Antenna Designs with different Migration Variants

\begin{tabular}{|c|c|c|c|c|c|c|c|c|}
\hline & \multicolumn{2}{|c|}{ Standard BBO } & \multicolumn{2}{c|}{ Blended BBO } & \multicolumn{2}{c|}{ IRBBO } & \multicolumn{2}{c|}{ EBBO } \\
\hline Element & Length & Spacing & Length & Spacing & Length & Spacing & Length & Spacing \\
\hline $1(\lambda)$ & 0.4815 & - & 0.4831 & - & 0.4840 & - & 0.4863 & - \\
\hline $2(\lambda)$ & 0.4760 & 0.2821 & 0.4765 & 0.2552 & 0.4647 & 0.2818 & 0.4896 & 0.2487 \\
\hline $3(\lambda)$ & 0.4470 & 0.2064 & 0.4420 & 0.2160 & 0.4458 & 0.1856 & 0.4425 & 0.2153 \\
\hline $4(\lambda)$ & 0.4293 & 0.3504 & 0.4277 & 0.3722 & 0.4294 & 0.3512 & 0.4285 & 0.3766 \\
\hline $5(\lambda)$ & 0.4172 & 0.4274 & 0.4192 & 0.4090 & 0.4172 & 0.4541 & 0.4217 & 0.3741 \\
\hline $6(\lambda)$ & 0.4090 & 0.4360 & 0.4113 & 0.4465 & 0.4126 & 0.4135 & 0.4110 & 0.4574 \\
\hline $7(\lambda)$ & 0.4080 & 0.4467 & 0.4078 & 0.4256 & 0.4065 & 0.4611 & 0.4087 & 0.4285 \\
\hline $8(\lambda)$ & 0.4036 & 0.4395 & 0.3967 & 0.4873 & 0.4051 & 0.4377 & 0.3984 & 0.4830 \\
\hline $9(\lambda)$ & 0.3962 & 0.5214 & 0.4006 & 0.5149 & 0.3995 & 0.4973 & 0.3987 & 0.4979 \\
\hline $10(\lambda)$ & 0.4048 & 0.4195 & 0.4043 & 0.4050 & 0.3998 & 0.4573 & 0.3964 & 0.4408 \\
\hline $11(\lambda)$ & 0.3952 & 0.4779 & 0.4009 & 0.4506 & 0.4008 & 0.4411 & 0.3964 & 0.4613 \\
\hline $12(\lambda)$ & 0.3977 & 0.4467 & 0.3927 & 0.4651 & 0.3963 & 0.4677 & 0.3950 & 0.4992 \\
\hline $13(\lambda)$ & 0.3949 & 0.4818 & 0.3973 & 0.5172 & 0.4008 & 0.4693 & 0.4055 & 0.4420 \\
\hline $14(\lambda)$ & 0.4000 & 0.4835 & 0.4096 & 0.3984 & 0.4032 & 0.4535 & 0.3950 & 0.4442 \\
\hline $15(\lambda)$ & 0.4141 & 0.3984 & 0.4187 & 0.4404 & 0.4127 & 0.4245 & 0.4183 & 0.4323 \\
\hline Gain $(\mathbf{d B i})$ & \multicolumn{2}{|c|}{$\mathbf{1 8 . 4 2}$} & \multicolumn{18}{|c|}{} & & $\mathbf{1 8 . 4 4}$ & $\mathbf{1 8 . 3 8}$ \\
\hline
\end{tabular}

design is optimized with 30 habitats and 500 iterations. Average of 10 monte-carlo simulations for each migration operator is presented here for fair comparative study of stochastic algorithm variants, viz. standard migration, blended migration, immigration refusal and EBBO as depicted in Figure 3. The C++

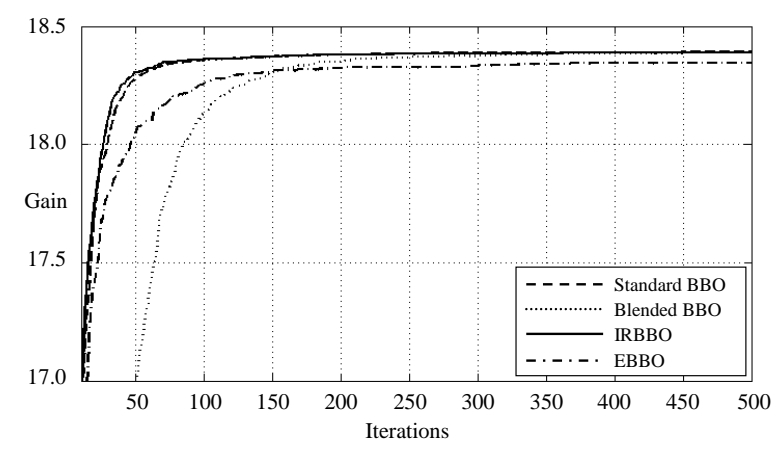

Fig. 3. Convergence Performance of BBO with Migration Options

programming platform is used for coding of $\mathrm{BBO}$ algorithm, whereas, a NEC2 [6] is used for evaluation of antenna designs based on method of moments. Each potential solution in BBO is encoded as vector with 29 SIVs as given by (1). The universe of discourse for the search of optimum values of wire-lengths and wire-spacings are $0.35 \lambda-0.50 \lambda$ and $0.10 \lambda-0.55 \lambda$, respectively, however, cross sectional radius and segment sizes are kept same for all elements, i.e., $0.003397 \lambda$ and $0.1 \lambda$ respectively, where $\lambda$ is the wavelength corresponding to frequency of operation, i.e, $300 \mathrm{MHz}$. Typically, the best antenna designs obtained during process of optimization are tabulated in Table 1.

From Figure 3, it can be seen that blended migration option yields the poorest solutions in initial iterations and later improves significantly than EBBO. Immigration refusal and standard migration variants are the fastest converging migration variants with immigration refusal option giving slightly better convergence performance than standard migration option. It can be concluded that immigration refusal is the best migration variant in term of convergence performance for Yagi-Uda antenna design problem.
It can be seen from Table 1 that IRBBO gives the highest and EBBO provides the lowest gain among all migration variants. Standard $\mathrm{BBO}$ and blended $\mathrm{BBO}$ gives the mediocre performance with standard $\mathrm{BBO}$ yielding higher gain than blended BBO.

\section{CONCLUSIONS AND FUTURE SCOPE}

In this paper, BBO algorithm is applied to optimize fifteenelement Yagi-Uda antenna designs for gain maximization. Here, BBO is experimented with different migration variants, viz., (1) Standard migration, (2) Blended migration, (3) Immigration Refusal and (4) EBBO, along with standard mutation operator. Here, it is observed that immigration refusal migration operator is the best option for optimizing the antenna designs. Investigation of BBO algorithms for other types of antenna designs is next on our agenda.

\section{REFERENCES}

[1] E.E. Altshuler and D.S. Linden. Wire-antenna Designs using Genetic Algorithms. Antennas and Propagation Magazine, IEEE, 39(2):33-43, 1997.

[2] A.N. Amaral, U.C. Resende, and E.N. Gonçalves. Yagi-uda antenna optimization by elipsoid algorithm. pages 503506, 2011.

[3] A.Wallace. The Geographical Distribution of Animals. Boston, MA: Adamant Media Corporation, Two:232-237, 2005.

[4] S. Baskar, A. Alphones, P N Suganthan, and J J Liang. Design of Yagi-Uda Antennas using Comprehensive Learning Particle Swarm Optimisation. IEEE, 152(5):340-346, 2005.

[5] JH Bojsen, H. Schjaer-Jacobsen, E. Nilsson, and J. Bach Andersen. Maximum Gain of Yagi-Uda Arrays. Electronics Letters, 7(18):531-532, 1971.

[6] G. J. Burke and A. J. Poggio. Numerical Electromagnetics Code (NEC) method of moments. NOSC Tech. DocLawrence Livermore National Laboratory, Livermore, Calif, USA, 116:1-131, 1981. 
[7] C. Chen and D. Cheng. Optimum Element Lengths for Yagi-Uda Arrays. IEEE Transactions on Antennas and Propagation, 23(1):8-15, 1975.

[8] D. Cheng and C. Chen. Optimum Element Spacings for Yagi-Uda Arrays. IEEE Transactions on Antennas and Propagation, 21(5):615-623, 1973.

[9] D. K. Cheng. Optimization Techniques for Antenna Arrays. Proceedings of the IEEE, 59(12):1664-1674, 1971.

[10] D. K. Cheng. Gain Optimization for Yagi-Uda Arrays. Antennas and Propagation Magazine, IEEE, 33(3):42-46, 1991.

[11] D. Correia, A. J. M. Soares, and M. A. B. Terada. Optimization of gain, impedance and bandwidth in Yagi-Uda Antennas using Genetic Algorithm. IEEE, 1:41-44, 1999.

[12] C. Darwin. The Orign of Species. New York : gramercy, Two:398-403, 1995

[13] D. Du, D. Simon, and M. Ergezer. Biogeography-based Optimization Combined with Evolutionary Strategy and Immigration Refusal. IEEE, 1:997-1002, 2009.

[14] H. Ehrenspeck and H. Poehler. A New Method for Obtaining Maximum Gain from Yagi Antennas. IRE Transactions on Antennas and Propagation, 7(4):379-386, 1959.

[15] R. M. Fishenden and E. R. Wiblin. Design of Yagi Aerials. Proceedings of the IEE-Part III: Radio and Communication Engineering, 96(39):5, 1949.

[16] E. A. Jones and W. T. Joines. Design of Yagi-Uda Antennas using Genetic Algorithms. IEEE Transactions on Antennas and Propagation,, 45(9):1386-1392, 1997.

[17] Y. Kuwahara. Multiobjective Optimization Design of YagiUda Antenna. IEEE Transactions on Antennas and Propagation, 53(6):1984-1992, 2005.

[18] J. Y. Li. Optimizing Design of Antenna using Differential Evolution. IEEE, 1:1-4, 2007.

[19] J.Y. Li. A bi-swarm optimizing strategy and its application of antenna design. Journal of Electromagnetic Waves and Applications, 23(14-15):1877-1886, 2009.

[20] Y. Li, F. Yang, J. OuYang, and H. Zhou. Yagi-uda antenna optimization based on invasive weed optimization method. Electromagnetics, 31(8):571-577, 2011.

[21] H. Ma and D. Simon. Blended Biogeography-based Optimization for Constrained Optimization. Engineering Applications of Artificial Intelligence, 24(3):517-525, 2011.

[22] R.H. MacArthur and E.O. Wilson. The Theory of Island Biogeography. Princeton Univ Pr, 1967.
[23] T. McTavish and D. Restrepo. Evolving Solutions: The Genetic Algorithm and Evolution Strategies for Finding Optimal Parameters. Applications of Computational Intelligence in Biology, 1:55-78, 2008.

[24] S. S. Pattnaik, M. R. Lohokare, and S. Devi. Enhanced Biogeography-Based Optimization using Modified Clear Duplicate Operator. IEEE, 1:715-720, 2010.

[25] M. Rattan, M. S. Patterh, and B. S. Sohi. Optimization of Yagi-Uda Antenna using Simulated Annealing. Journal of Electromagnetic Waves and Applications, 22, 2(3):291299, 2008.

[26] D. G. Reid. The Gain of an Idealized Yagi Array. Journal of the Institution of Electrical Engineers-Part IIIA: Radiolocation, , 93(3):564-566, 1946.

[27] L. C. Shen. Directivity and Bandwidth of Single-band and Double-band Yagi Arrays. IEEE Transactions on Antennas and Propagation, 20(6):778-780, 1972.

[28] D. Simon. Biogeography-based Optimization. IEEE Transactions on Evolutionary Computation,, 12(6):702-713,

[29] U. Singh, H. Kumar, and T. S. Kamal. Design of Yagi-Uda Antenna Using Biogeography Based Optimization. IEEE Transactions on Antennas and Propagation, , 58(10):3375$3379,2010$.

[30] U. Singh, M. Rattan, N. Singh, and M. S. Patterh. Design of a Yagi-Uda Antenna by Simulated Annealing for Gain, Impedance and FBR. IEEE, 1:974-979, 2007.

[31] Shintaro Uda and Yasuto Mushiake. Yagi-Uda Antenna. Maruzen Company, Ltd, 1954.

[32] N. V. Venkatarayalu and T. Ray. Single and Multi-Objective Design of Yagi-Uda Antennas using Computational Intelligence. IEEE, 2:1237-1242, 2003.

[33] N.V. Venkatarayalu and T. Ray. Optimum Design of YagiUda Antennas Using Computational Intelligence. IEEE Transactions on Antennas and Propagation,, 52(7):18111818,2004

[34] H. J. Wang, K. F. Man, C. H. Chan, and K. M. Luk. Optimization of Yagi array by Hierarchical Genetic Algorithms. IEEE, 1:91-94, 2003.

[35] H. Yagi. Beam Transmission of Ultra Short Waves. Proceedings of the Institute of Radio Engineers, 16(6):715$740,1928$. 